\title{
DUAL-SOURCE COMPUTED TOMOGRAPHY CORONARY ARTERY IMAGING IN CHILDREN
}

\author{
Aurelio Secinaro, Davide Curione, Kristian Havmand Mortensen, Teresa Pia \\ Santangelo, Paolo Ciancarella, Carmela Napolitano, Alessia Del Pasqua, Andrew \\ Mayall Taylor \& Paolo Ciliberti
}

Pediatric Radiology 2019; 49: 1823-1839

\begin{abstract}
Computed tomography (CT) has a well-established diagnostic role in the assessment of coronary arteries in adults. However, its application in a pediatric setting is still limited and often impaired by several technical issues, such as high heart rates, poor patient cooperation, and radiation dose exposure. Nonetheless, CT is becoming crucial in the non-invasive approach of children affected by coronary abnormalities and congenital heart disease. In some circumstances, CT might be preferred to other noninvasive techniques such as echocardiography and magnetic resonance imaging for its lack of acoustic window influence, shorter acquisition time, and high spatial resolution.

The introduction of dual source computed tomography (DSCT) has expanded the role of $\mathrm{CT}$ in the evaluation of pediatric cardiovascular anatomy and pathology. Furthermore, technical advances in the optimization of low-dose protocols represent an attractive innovation. DSCT can play a key role in several clinical settings in children, namely in the evaluation of patients with suspected congenital coronary artery anomalies, both isolated and in association with congenital heart disease. Moreover, it is able to assess acquired coronary artery abnormalities, as in patients with Kawasaki
\end{abstract}


disease and after surgical manipulation, especially in case of transposition of the great arteries treated with arterial switch operation and in case of coronary reimplantation.

Keywords: Dual Source Computed Tomography, Coronary Artery Imaging, Coronary Artery Anomalies, Congenital Heart Disease 


\section{INTRODUCTION}

Coronary artery imaging is often challenging in children due to problems related to small size, high heart rates, motion artifacts from cardiac movement and respiration, and limited patient cooperation. Therefore, high spatial and temporal resolution, short examination time and low patient risks are required [1].

Imaging modalities for evaluating coronary arteries in children include echocardiography, conventional invasive angiography, magnetic resonance imaging (MRI), and computed tomography (CT). Transthoracic echocardiography is widely used as the primary imaging approach [2]. However, it is impaired by its limited ability to fully characterize coronary anatomy, by poor acoustic windows, and by operatordependency. Conventional angiography can provide both anatomical and functional information, particularly in patients with congenital heart disease, but is associated with non-negligible risks related to its intrinsic invasiveness and to the use of potentially high doses of ionizing radiation and iodinated contrast agent [3]. Thus, it should be reserved for interventional procedures or when noninvasive diagnostic imaging is inconclusive. MRI also provides information on cardiac anatomy and function, allowing 3-dimensional coronary artery imaging without the use of ionizing radiation. However, it is impaired by long acquisition time requiring prolonged patient cooperation, which may not be possible in children without general anesthesia, and by

limited spatial resolution [4], which makes coronary evaluation beyond origin assessment difficult.

CT is currently considered as the diagnostic method of choice for noninvasive coronary artery imaging. Recently, it has undergone significant advances in the technical field and, especially with the advent of dual source CT (DSCT) and modern strategies to reduce radiation dose, it has been used more frequently to evaluate 
coronary arteries and complex congenital heart disease in children [5]. At present, several congenital and acquired coronary artery abnormalities can be accurately and safely described by $\mathrm{CT}$ in the pediatric population.

\section{TECHNICAL ASPECTS}

Each cardiac CT exam should be tailored to the individual patient in order to maximize diagnostic information and minimize risk. Compared to most other indications in congenital heart disease, optimal coronary artery imaging requires higher image quality, implying the highest spatial and temporal resolution available, possibly associated with slow and regular heart rate and breath holding [6]. State-of-the-art second and third-generation DSCT scanners (SOMATOM Definition Flash ${ }^{\circledR}$ and SOMATOM Force ${ }^{\circledR}$ respectively, Siemens Healthineers, Erlangen, Germany; Table 1) allow for submillimeter isotropic imaging of the heart and coronary arteries in one or few heart beats, paired with dose reduction techniques including high-pitch spiral scanning, low tube voltage acquisition, and iterative reconstruction [7]. In our center, we perform all CT exams using a second-generation DSCT scanner.

\section{Patient preparation}

Children should be carefully positioned on the CT table at the isocenter of the scanner in the supine position, with the arms above the head and with ECG electrodes outside the thoracic region, on the arms and upper abdomen, to prevent to prevent image quality deterioration (i.e. beam hardening artifacts) or radiation dose increase [8]. 
Thanks to the versatility of DSCT, the majority of exams can be performed without sedation/anesthesia. Although minor motion artifacts may not significantly alter image quality [9], it is important to ensure patients remain still during scanning. If they are unable to cooperate or agitated, various solutions can be adopted [10].

Mostly, only information on proximal or anatomical course of the coronary arteries is requested in children. Therefore, they may be scanned free-breathing if unable to follow instructions [11], even at high heart rates, since there is limited motion of the proximal coronary vessels throughout the cardiac cycle. Free-breathing scanning is one the most notable advantages of DSCT. However, when distal or detailed coronary artery imaging is needed, breath holding is beneficial. If patients are compliant, practicing the breath hold before the exam helps to maximize cooperation and to assess respiratory sinus arrhythmia. Otherwise, anesthesia with suspended respiration may be considered [6]. For the same reasons, nitrates and/or beta-blockers are often not needed to evaluate the coronary arteries in children, given they prolong patient preparation time with no guaranteed success [12]. Nevertheless, they have been shown to be safe in children [13] with careful screening for contraindications and should be considered $[14,15]$ if high definition imaging is required, particularly beta-blockers, since image quality and radiation reduction algorithms remain heart-rate dependent.

\section{Contrast injection}

Iodinated contrast agent is usually injected via a peripheral vein using a dualsyringe power injector [16]. Intravenous line size is affected by the site of cannulation and body size, typically ranging from 24 gauges $(\mathrm{G})$ in neonates to $18 \mathrm{G}$ in older children, with flow rates between 0.5 and $>5 \mathrm{~mL} / \mathrm{s}$ [17] and pressure settings between 50 and 300 pounds per square inch (psi). Central venous catheters may also be used for 
contrast infusion, especially in critically ill patients, but they may only allow for adequate injection in younger children as they generally have lower maximum flow rates and psi values [18] (0.4-1.2 mL/s and 25-50 psi), or may require a hand injection.

Contrast concentration and volume should be adapted to the intravenous access and body weight, employing 300-400 $\mathrm{mgI} / \mathrm{mL}$ agents at $1-3 \mathrm{~mL} / \mathrm{kg}$ until standard adult contrast amounts are reached. Lower concentrations with higher volumes are typically used in infants and younger children with smaller intravenous lines or in case of central venous catheters, whereas higher concentrations with lower volumes are usually infused in older children and adolescents with larger cannulas (Table 2). Special care must be taken to avoid accidental injection of air, which may result in paradoxical embolism in the presence of intra-cardiac shunting.

The most common injection protocols include a biphasic and triphasic approach [6]. The biphasic protocol consists in contrast administration followed by a saline bolus, timing image acquisition to opacification of the ascending aorta. This is the usual method for coronary artery imaging if there are no associated cardiovascular lesions. The triphasic protocol entails a two-phase contrast infusion (about half at the regular rate plus the remainder either at a slower rate or as a contrast:saline mix) [19] followed by a saline flush. It is most frequently used in the presence of associated cardiovascular lesions to obtain simultaneous evaluation of right and left-sided structures.

\section{Scan timing}

On the one hand, a fixed time can be set to initiate scanning at the end of the injection protocol (typically about $20 \mathrm{~s}$ ). The site of cannulation and scan protocol should be considered, with additional delay when injecting from the leg and earlier acquisition when using longer scan methods. This approach may be best suitable for 
infants and younger children, as it avoids unnecessary radiation exposure related to the use of bolus monitoring scans. Besides, for many indications contrast opacification need not be as high as in conventional adult coronary imaging. Nevertheless, this method is challenging, because it may be prone to inaccuracy and limited reproducibility due to the variability of contrast transit time for technical and anatomical/functional reasons, particularly in patients with single ventricle physiology, valvular pathology or ventricular dysfunction [6].

On the other hand, bolus monitoring techniques enable to perform scanning at optimal opacification time and are especially useful in older children with higher intervariability [17]. The test bolus approach consists in administering a small amount of contrast and calculating vascular peak enhancement time by monitoring contrast transit on a target slice. An extra delay (usually 3-6 s) must be added in the actual acquisition depending on various factors (e.g. body size, scan protocol) [20]. Since this method involves both additional contrast and radiation exposure, it is not routinely used in children. With the bolus tracking technique, the scan is triggered when vascular enhancement reaches an adequate level in a structure of interest monitored on a target slice [21]. Automatic triggering, using a region of interest with a Hounsfield unit (HU) threshold (e.g. $150 \mathrm{HU}$ at $100 \mathrm{kV}$, with higher thresholds for lower $\mathrm{kV}$ ), may be difficult in children, given the risk of errors due to the combination of small vascular structures and potential movement. Manual initiation with a visual approach more reliably ensures ideal opacification, especially in the context of complex congenital heart disease where contrast may not follow the expected route. To reduce radiation exposure, monitoring should start near the end of contrast injection, should be reduced in frequency, and both tube power and current should be decreased [10]. 


\section{Scan protocol}

The scan range should include the heart for standard coronary imaging but may need to be extended to the ascending aorta/pulmonary arteries or further depending on the clinical indication, especially in the context of congenital heart disease.

The main acquisition methods include prospective ECG-triggered high-pitch dual-source spiral, prospective ECG-gated sequential and retrospective ECG-gated spiral scanning.

Prospective ECG-triggered high-pitch dual-source spiral scanning [(Turbo) Flash], unique to DSCT, uses both x-ray tubes within the scanner to achieve both maximum temporal resolution and z-axis scan speed, resulting in rapid and large gapless non-overlapping imaging in a single heart beat with a limited field-of-view (corresponding to that of the secondary tube). Data acquisition is prospectively triggered by the ECG so that subsequent images are obtained at progressively later times within the cardiac cycle [22]. This means the dataset is not uniform in time and cannot be ECG-edited, contrary to sequential and retrospective ECG-synchronized scans. This method can provide accurate evaluation of the whole coronary tree using diastolic triggering in patients with regular heart rates below 60-65 bpm. Nevertheless, it can also be used at higher heart rates with end-systolic triggering to visualize proximal coronary course, although motion artifacts may occur especially at the end of the scanned volume. Additionally, it is particularly recommended in case of uncooperative patients, because it reduces respiratory and cardiac motion artifacts, as well as the need for sedation and general anesthesia [11]. When there is need for extended anatomic coverage in combination with coronary assessment, it may be appropriate to use a protocol that allows to scan a large volume in the high-pitch modality with a prospective ECG-triggered box targeted at the level of the heart or, if 
only proximal coronary course is required, at the level of the aortic root (Fig. 1). High accuracy in the evaluation of intracardiac structures and coronary arteries with only minor image quality deterioration has been reported for the high-pitch acquisition [5]. Several studies in the literature have shown lower radiation doses compared to the prospective ECG-gated sequential method, with a mean effective radiation dose of about $0.2-0.5 \mathrm{mSv}[23,24]$.

In prospective ECG-gated sequential (step-and-shoot) and retrospective ECGgated spiral scanning, imaging occurs with acquisition of a definite portion or all of the $\mathrm{R}-\mathrm{R}$ interval respectively, in order to find the best resting period for each coronary artery [17]. Both x-rays tubes are still employed to achieve maximum temporal resolution over multiple heart beats without field-of-view limitation. The sequential method is especially recommended for detailed assessment of the coronary tree, particularly in cooperative patients with higher or irregular heart rates. A drawback is the possibility of stack artifacts, which however seldom seriously affect image interpretation in children. Prospective scanning still entails low radiation dose, especially when compared to retrospective scanning, with a mean effective dose around 0.3 up to $1 \mathrm{mSv}[17,25]$. Besides, the high temporal resolution of DSCT has drastically reduced the need for the retrospective technique, particularly for high-dose protocols with multi-segment reconstruction, which are necessary at high heart rates with single source scanners. Moreover, although retrospective scanning can currently be obtained at relatively low radiation dose (mean effective dose approximately $2-5 \mathrm{mSv}$ ) $[17,26]$ thanks to several strategies, namely the use of intensive pulsing modulation and higher pitch values at higher heart rates, it should be considered in selected cases, especially when looking for detailed coronary imaging with challenging elevated and highly 
irregular heart rhythm or when seeking dynamic information, such as valvular motion or ventricular function [10]. Fig. 2 outlines suggestions for protocol selection.

All CT examinations should employ a low-dose protocol, including weightadapted settings for tube voltage $[80 \mathrm{kV}$ below $40 \mathrm{~kg}$ (consider $70 \mathrm{kV}$ in neonates/infants), $100 \mathrm{kV}$ above $40 \mathrm{~kg}$ (consider $120 \mathrm{kV}$ in overweight/high-density objects)] and current (10 mAs/kg below $5 \mathrm{~kg}$, then 4-5 mAs/kg up to $92 \mathrm{mAs}$ between 6-15 kg, then 2-3 mAs/kg up to $138 \mathrm{mAs}$ above $15 \mathrm{~kg}$ ) [27]. Another strategy to reduce radiation dose is (semi-)automatic modulation of tube voltage and current [5,28].

Compared to other state-of-the-art technology, namely 256- and 320-row scanners, DSCT has unparalleled temporal resolution (66-75 ms versus at least $140 \mathrm{~ms}$ ), resulting in motion-free imaging even at the high heart rates of children. On the other hand, 256- and 320-row machines are capable of greater z-axis coverage in one gantry rotation (160 mm versus 38.4-57.6 mm of DSCT), allowing for acquisition of the whole heart in a single heartbeat, thus reducing misregistration artifacts and contrast volume [29]. This can also be achieved by DSCT in the prospective high pitch mode, even if low heart rates are required for high definition imaging. Finally, radiation dose is similar among scanners $[30,31]$, though with lowest values for the high pitch technique.

\section{Image reconstruction and interpretation}

DSCT raw data can be reconstructed using two techniques, filtered back projection and iterative reconstruction. The former is the standard analytical CT method, while the latter is a noise-reducing algorithm that employs measured raw and simulated data to generate image estimates which are compared and corrected in cycles at different strength levels. The major benefit of iterative reconstruction is the possibility to reduce image noise in exams acquired with low-dose protocols, 
preserving image quality [32]. Also, artifacts typically associated with filtered back projection are decreased, such as beam hardening or blooming artifacts arising from high-density objects like coronary stents, allowing for better delineation of vessel lumen. The combination of iterative reconstruction and low-tube voltage enables to decrease the volume of contrast agent without image quality deterioration [27].

In prospective sequential or retrospective spiral scans, the best cardiac phase for image reconstruction can be automatically defined by the scanner, thanks to software approaches based on motion maps applied to the raw data set for automatic determination of minimal cardiac motion. If unsatisfactory, the best cardiac phase may be manually decided by the operator through multiphase evaluation, in which case it is important to reconstruct several phases to determine the best frame of cardiac rest, keeping in mind that multiple phases may be needed to fully assess the different portions of the coronary tree. In case of irregular heart rate, it may be advisable to reconstruct phases according to milliseconds rather than relative percentage intervals, as this method may be superior [33]. In addition to transaxial images, the following reconstructions are recommended for image interpretation [34,35]: multiplanar reformations in three adjustable planes, including curved planar reformations along the coronaries to display their tortuous course and vessel cross sections for lumen evaluation when necessary; maximum intensity projection images for "angiographylike" views, allowing quick and intuitive visualization of coronary course; volume rendering reconstructions for a three-dimensional overview of coronary anatomy, including virtual angioscopy that may be useful to depict the coronary ostia. All these steps should be performed by clinicians (radiologists in our center) and may be timeconsuming, especially in case of high definition imaging and/or complex congenital heart disease (up to 30 minutes to 1 hour). 


\section{CLINICAL INDICATIONS}

Coronary artery abnormalities in children may be congenital or acquired. Congenital anomalies include a complex group of disorders occurring as isolated conditions or in the spectrum of congenital heart disease. Acquired coronary anomalies are mainly secondary to Kawasaki disease or surgery when congenital heart disease repair involves coronary manipulation. Increasing evidence is supporting the role of CT in general and DSCT in particular for coronary evaluation of these patients [36,37].

\section{CONGENITAL CORONARY ARTERY ANOMALIES}

\section{Isolated anomalies}

Isolated coronary abnormalities are the second most common cause of cardiovascular death in young athletes in Europe and the United States [38]. Clinical manifestations vary, ranging from incidental findings to sudden cardiac death ("malignant" variants). Three main categories can be identified, including anomalies of origin and course, of intrinsic coronary anatomy and of termination (Table 3) [39].

Anomalies of origin and course entail variation in the number, shape and location of the coronary ostia. On the one hand, benign variants are quite common and, although not hemodynamically significant, they may become relevant in case of cardiac surgery. On the other hand, some abnormalities are associated with high mortality. In particular, anomalous origin of the left coronary artery from the pulmonary artery (Fig. 3), albeit a rare condition (1:300.000 births), can cause a coronary steal phenomenon into the pulmonary circulation with development of intercoronary collaterals, leading 
to myocardial ischemia, heart failure and ventricular arrhythmias since the early neonatal period [40]. A less dramatic but still potentially fatal condition is inappropriate origin of a coronary artery with interarterial and/or intramural course (Figs. 4 and 5), particularly dangerous if involving the left coronary artery. This pattern is often asymptomatic, but sudden death particularly during maximal exercise can occur. CT has a key role in these settings, for confirming the diagnosis, for aiding in surgical planning when indicated and for ruling out complications after a corrective operation [41]. Specifically, CT may be superior to conventional angiography in defining the ostial origin and proximal course of anomalous coronary branches [42], providing 3D anatomical information that may be difficult to obtain with invasive angiography and avoiding potential issues related to cannulation of anomalous coronary vessels.

Myocardial bridging is a frequently encountered intrinsic coronary anomaly (frequency near $25 \%$, range $5-86 \%$ in autoptic and imaging studies) [43] whereby a coronary artery segment shows intramuscular rather than epicardial course. All coronary tracts can be involved, but the middle left anterior descending artery seems to be the most common [4]. In myocardial bridging, the affected vascular segment can become compressed during systole, sometimes creating transitory myocardial ischemia. It has been advocated that most instances are of little clinical importance. However, several reports suggest that some cases may produce coronary thrombosis, myocardial ischemia, and infarction, as well as predispose to atherosclerosis or sudden death $[45,46]$. In general, myocardial bridging should be suspected in patients with exertional angina but no coronary risk factors, especially if young. Compared to catheter angiography, CT clearly shows the intramyocardial location of the involved coronary segment (Fig. 6). Obtaining both systolic and diastolic phases allows assessment of luminal narrowing throughout the cardiac cycle [42]. 
Anomalies of coronary termination essentially comprise coronary artery fistulas, which are communications of one or more coronary arteries, more commonly the right coronary artery, with a cardiac chamber, usually right-sided structures such as the right ventricle, right atrium, pulmonary artery and coronary sinus. Coronary fistulas are very uncommon, being found in only $0.15 \%$ of patients undergoing conventional angiography [47]. They are generally due to embryological development disturbances but may also be acquired after trauma or invasive cardiac procedures [36]. The sites of origin and termination as well as the size of the abnormal connection have an impact on clinical significance. Most fistulas are small without clinical implications, but large connections may entail significant left-to-right shunt and coronary artery steal, which can lead to ischemia of the portion of myocardium perfused by the coronary segment distal to the fistula. When present, clinical manifestations include chest pain and dyspnea in adults, while children are often asymptomatic. CT can provide 3Danatomical images that help to confirm the diagnosis and better delineate the fistulous communications (Fig. 7), facilitating treatment planning as well as the detection of post-treatment complications and potentially acting as a gatekeeper to invasive angiography, which could mainly be reserved for treatment [41].

\section{Anomalies associated with congenital heart disease}

Coronary artery abnormalities are more common in patients with congenital heart disease. Even when clinically irrelevant, these lesions may become important as they may affect surgical repair [48]. Unambiguous coronary artery imaging is therefore mandatory in this situation and, especially when echocardiography is not conclusive, CT should be employed [49] to avoid unnecessary invasive procedures, even in the 
neonatal period. The most common conditions to consider in this setting include tetralogy of Fallot and (dextro-)transposition of the great arteries.

Tetralogy of Fallot is the most common cyanotic cardiac defect, defined by the classic tetrad of ventricular septal defect, overriding aorta, pulmonary outflow tract stenosis or atresia and right ventricular hypertrophy. Complete repair involves ventriculotomy at the level of the right ventricular outflow tract. Consequently, ruling out an epicardial coronary vessel in that area prior to surgery is crucial. The most important abnormalities to recognize are anomalous origin of the left anterior descending artery from the right coronary artery with prepulmonary course, and a double left anterior descending artery, usually consisting in a short proximal vessel arising from the left coronary and a longer distal vessel arising from the right coronary that crosses the right outflow [50] (Fig. 8). Additionally, coronary artery anatomy should be known before repeat intervention on the outflow [51], repeat sternotomy to rule out substernal coronary course or transcatheter pulmonary valve placement to prevent coronary compression [49].

Transposition of the great arteries represents $5-7 \%$ of all forms of congenital heart disease and is characterized by ventriculo-arterial discordance, with origin of the aorta from the right ventricle and origin of the pulmonary trunk from the left ventricle, leading to cyanosis as a result of two parallel circulations. Great vessel and coronary anatomy may be variable and have several classification systems [52]. Most commonly, the aorta is anterior and rightward relative to the pulmonary artery. Aortic sinuses are often described based on whether they are adjacent to the pulmonary artery, almost always bearing the coronary ostia, or not as facing and nonfacing, respectively. The most frequent or "usual" coronary pattern involves the left coronary artery arising from the left anterior facing sinus and the right coronary artery originating from the right 
posterior facing sinus. Other notable arrangements include the left circumflex artery arising from the right coronary with a retropulmonary course (second most common), the left coronary coming off the right posterior facing sinus with a retropulmonary course and the right coronary originating from the left anterior facing sinus ("inverted" pattern), and single coronary artery [53]. Alternative classification schemes have been recently proposed where the aortic sinuses and coronary ostia are defined according to their relative location to the patient's axial anatomy and sequentially described [52]. Rarely, coronary course may be intramural and/or interarterial (Fig. 9). Native coronary anatomy is a risk factor for the development of complications after surgical repair, particularly in patients with single coronary orifice or intramural/interarterial course, who have significantly increased mortality and higher rate of late adverse events [54].

\section{ACQUIRED CORONARY ARTERY ANOMALIES}

\section{Kawasaki disease}

Kawasaki disease is a febrile systemic vasculitis of unknown origin that usually occurs in infancy and childhood. The acute phase is characterized by high fever, cutaneous rash, non-exudative conjunctivitis, oral mucosa inflammation, cervical adenopathy, swollen hands and feet, red palms and soles, and subungual peeling [55]. Acute cardiac manifestations include pericarditis, myocarditis, heart valve dysfunction and arrhythmias. About $15-25 \%$ of patients, especially when not treated early with high-dose intravenous immunoglobulins, can develop coronary artery aneurysms, which may undergo thrombosis and progressive stenosis causing myocardial ischemia, infarction, and sudden death [56]. Coronary aneurysms are more commonly located along the proximal left and right coronary arteries, but any segment can be affected. They can be saccular, fusiform, or there can be diffuse ectasia without segmental 
dilation. They can evolve over time in shape and size, increasing in dimension over the first 1-2 months after the onset of the disease and regressing in up to two thirds of cases to normal lumen diameter within 2 to 5 years [57], with further regression being unlikely. Therefore, imaging follow-up of the coronary arteries is essential in these patients. Echocardiography has high diagnostic accuracy for proximal aneurysms, but it is less sensitive for distal lesions and coronary artery stenosis, which usually worsens over time in the affected segments. For these reasons, CT is widely used to obtain highresolution imaging of the whole coronary tree [4,58] (Figs. 10 and 11), offering comparable accuracy to conventional angiography [59]. In case of giant aneurysms, repeating the scan at equilibrium contrast-phase may help to differentiate thrombosis from flow artifacts and depict persistent myocardial perfusion defects (Fig. 12).

\section{Post-operative status}

Different surgical procedures employed for congenital coronary anomalies and heart disease entail coronary artery manipulation, leading to possible complications in the early and late post-operative course. In the setting of congenital heart disease, the most common interventions involving coronary reimplantation include the arterial switch operation for treatment of transposition of the great arteries and the Ross operation for management of aortic valve disease.

Since the early ' 80 s, the arterial switch operation has become the standard corrective technique for transposition in the absence of significant associated lesions like pulmonary stenosis [60]. The procedure recreates physiological ventriculo-arterial concordance by transecting the great arteries and translocating them to the opposite root, with rearrangement of the pulmonary arteries anterior to the aorta (Lecompte maneuver). Translocation of the aorta implies mobilization and reimplantation of the 
coronary arteries, which are excised with a small cuff of aortic wall to be resutured into the neo-aortic root, with a somewhat stretched course compared to their original position (Fig. 13). In the early experiences, coronary manipulation was the main surgical issue, leading to coronary insufficiency and high perioperative mortality. Coronary transfer techniques have greatly improved since, with a substantial drop in hospital mortality, although the long-term success of the operation remains strictly dependent on the patency of the reimplanted coronary vessels. The majority of coronary events occur in the first three months after surgery [54] and are usually related to kinking or other types of anatomic obstruction to coronary perfusion. Late complications have an incidence of up to $10 \%$ and may lead to myocardial ischemia or infarction [61]. Imaging of coronary anastomoses is recommended in symptomatic patients, as well as at least once in asymptomatic patients during adolescence or early adulthood [51]. Although catheter angiography is still the gold standard for coronary lesions, CT currently represents a reasonable alternative (Fig. 14), providing highresolution imaging and reducing both the need and the risks of invasive studies, such as coronary ostium alteration during catheter placement [62].

The Ross or Ross-Konno procedure is an alternative to prosthetic valve replacement in children with aortic valve stenosis [63]. During surgery, the pulmonary valve is translocated to the aortic position and a homograft conduit is placed to reestablish continuity from the right ventricle to the pulmonary artery. As with the arterial switch operation, the coronaries need to be excised form the native aortic sinuses and reimplanted into the neo-aortic root, but complications are less frequent because the vessels are reattached almost in their native position rather than being stretched (Fig. 15). Over recent years, other surgical operations implying coronary manipulation, such as the Nikaidoh procedure for patients with transposition, 
ventricular septal defect and pulmonary stenosis, are becoming more common, thus expanding the potential indications for coronary artery follow-up by CT [49].

Finally, CT is useful to evaluate coronary artery bypass grafts and stents $\geq 3$ $m m$ in children with congenital or acquired heart disease undergoing revascularization [64] (Fig. 16) and to assess cardiac allograft vasculopathy in heart transplant patients [65]. CT has high sensitivity, specificity, and negative predictive values in these settings, comparable to invasive coronary angiography, and may be used during followup for detecting significant coronary artery lesions.

\section{CONCLUSIONS}

The technical improvements of DSCT represent a major advantage in the evaluation of children with coronary artery abnormalities, as they allow reliable assessment of small patients with high heart rates by using low-dose protocols that minimize cardiac and respiratory motion artifacts and limit the need for sedation/anesthesia. These benefits enable the use of DSCT as a key technique in the workup of both congenital and acquired coronary anomalies in children, simultaneously opening new frontiers to expand current clinical indications. 
TABLES

Table 1 Main technical specifications for second and third-generation DSCT scanners

\begin{tabular}{|l|r|r|}
\hline \multicolumn{1}{|c|}{ Characteristics } & $\begin{array}{c}\text { Second-generation } \\
\left.\text { (SOMATOM Definition Flash }{ }^{\circledR}\right)\end{array}$ & $\begin{array}{c}\text { Third-generation } \\
\left.\text { (SOMATOM Force }{ }^{\circledR}\right)\end{array}$ \\
\hline Number of detector rows & $128(64 \times 2)$ & $192(96 \times 2)$ \\
\hline Detector z-dimension & $0.6 \mathrm{~mm}$ & $0.6 \mathrm{~mm}$ \\
\hline Rotation time & $0.28 \mathrm{~s}$ & $0.25 \mathrm{~s}$ \\
\hline Temporal resolution & $75 \mathrm{~ms}$ & $66 \mathrm{~ms}$ \\
\hline Spatial resolution & $0.30 \mathrm{~mm}$ & $0.24 \mathrm{~mm}$ \\
\hline Z-axis coverage & $38.4 \mathrm{~mm}$ & $57.6 \mathrm{~mm}$ \\
\hline Max scan speed & $458 \mathrm{~mm} / \mathrm{s}($ Flash) & $737 \mathrm{~mm} / \mathrm{s}$ (Turbo Flash) \\
\hline Field-of-view & Main tube: $50 \mathrm{~cm}$ & Main tube: $50 \mathrm{~cm}$ \\
& Secondary tube: $33 \mathrm{~cm}$ & Secondary tube: $35 \mathrm{~cm}$ \\
\hline$k V$ settings & $70,80-140$ in steps of 20 & $70-150$ in steps of 10 \\
\hline Iterative reconstruction & SAFIRE & ADMIRE \\
\hline ADMIRE, Advanced Modeled Iterative Reconstruction; SAFIRE, Sinogram Affirmed Iterative Reconstruction.
\end{tabular}

Table 2 Recommendations for contrast administration

\begin{tabular}{|l|r|r|r|r|r|}
\hline \multicolumn{2}{|c|}{ Intravenous line } & \multicolumn{2}{c|}{ Power injection } & \multicolumn{2}{c|}{ Contrast } \\
\hline \multicolumn{1}{c|}{ Type } & Size & Flow rate & Pressure & Concentration & Volume \\
\hline Central & Any & $\leq 1.5 \mathrm{~mL} / \mathrm{s}$ & $\leq 50 \mathrm{psi}$ & & \\
\hline \multirow{4}{*}{ Peripheral } & $24 \mathrm{G}$ & $\leq 1.5 \mathrm{~mL} / \mathrm{s}$ & $50-100 \mathrm{psi}$ & $\leq 350 \mathrm{mgI} / \mathrm{mL}$ & $2-3 \mathrm{~mL} / \mathrm{kg}$ \\
\cline { 2 - 4 } & $22 \mathrm{G}$ & $\leq 3 \mathrm{~mL} / \mathrm{s}$ & $100-200 \mathrm{psi}$ & & \\
\cline { 2 - 4 } & $20 \mathrm{G}$ & $\leq 5 \mathrm{~mL} / \mathrm{s}$ & $300 \mathrm{psi}$ & \multirow{2}{*}{$\leq 400 \mathrm{mgI} / \mathrm{mL}$} & $1-2 \mathrm{~mL} / \mathrm{kg}$ \\
\cline { 2 - 4 } & $18 \mathrm{G}$ & $\leq 6.5 \mathrm{~mL} / \mathrm{s}$ & $300 \mathrm{psi}$ & & \\
\hline
\end{tabular}

Table 3 Main classes of congenital coronary artery anomalies 


\begin{tabular}{|c|c|}
\hline \multirow{3}{*}{$\begin{array}{c}\text { Anomalies of coronary } \\
\text { origin and course }\end{array}$} & $\begin{array}{l}\text { Anomalous coronary ostium outside of the aortic sinuses, } \\
\text { including anomalous origin of the left coronary artery from } \\
\text { the pulmonary artery }\end{array}$ \\
\hline & $\begin{array}{l}\text { Anomalous location of coronary ostium at improper sinus, } \\
\text { including interarterial/intramural course }\end{array}$ \\
\hline & Single coronary artery \\
\hline \multirow{6}{*}{$\begin{array}{l}\text { Anomalies of intrinsic } \\
\text { coronary anatomy }\end{array}$} & Congenital ostial stenosis or atresia \\
\hline & Coronary ectasia and aneurysm \\
\hline & Myocardial bridging \\
\hline & Duplicated arteries \\
\hline & Subendocardial coronary course \\
\hline & Coronary crossing \\
\hline $\begin{array}{c}\text { Anomalies of coronary } \\
\text { termination }\end{array}$ & Coronary artery fistulas \\
\hline
\end{tabular}




\section{FIGURE CAPTIONS}

Fig. 1 High-pitch spiral protocol with prospective ECG-triggered box. a Scanogram shows the large non-triggered chest volume (blue square) with the smaller prospective ECG-triggered box (red square) at the level of the aortic root. $b$ ECG report indicates the chest (blue square) and coronary (red square) acquisition windows with respect to the R-R interval. Coronary scan is set at end-systole, in accordance with the high heart rate (106 bpm).

Fig. 2 Suggestions for DSCT protocol selection for coronary artery imaging in children. There are three main stages. 1. The first step consists in assessing patient compliance. Various strategies can be employed in uncooperative patients to avoid general anesthesia: physical immobilization devices (i.e. soft weights, restraining bands, or vacuum cushion [10]); swaddling and scanning while sleeping, especially after eating ("feed-and-wrap" technique), oral glucose, or pacifiers in infants; parent/tutor or audio/video support in younger children. If necessary, mild sedation with short-acting benzodiazepines, such as midazolam, may be administered. 2 . The second step involves evaluating heart rate to determine the best cardiac phase for coronary imaging. In general, to ensure the heart is imaged during its optimal motion-free time frame, patients with lower heart rates (below 70-75 bpm) should be scanned during mid-tolate diastole (about $70 \%$ of the R-R interval), whereas patients with higher heart rates (above 70-75 bpm) should be scanned at end-systole (around $40 \%$ of the R-R interval) [66]. 3. Finally, the purpose of the exam guides protocol choice. Mostly, only information on proximal or anatomical course of the coronary arteries is requested in children. Prospective high-pitch spiral scanning can achieve this goal at low as well as high heart rates, although it may be less reliable and more indicated when coronary imaging is not the primary concern at elevated heart rhythms, especially with the ECGtriggered box at the level of the aortic root. High definition imaging with this technique requires low and stable heart rates. Prospective sequential scanning should be the preferred method whenever the high-pitch technique is not suitable. In contrast, retrospective spiral scanning should be reserved to cases where concurrent dynamic evaluation (e.g. ventricular function) is required. In these modalities, acquisition window (or ECG pulsing) refers to the portion of cardiac cycle in which the scanner acquires data for prospective scanning or delivers maximum tube current for 
retrospective scanning, respectively. If the heart rate is regular, a narrow acquisition window should be used [67], typically selected as a relative percentage interval of the cardiac cycle (5-10\%). In contrast, in patients with significant heart rate variability, a widened acquisition window (20-40\%) and/or millisecond scanning may be employed, making use of an adaptive algorithm of correction [68] that dynamically modifies the scanned portion of the cardiac cycle according to the frequency. Medications, namely beta-blockers and/or nitrates, are often not necessary for coronary artery assessment in children, but should be considered to achieve high definition imaging.

Fig. 3 1-year-old girl with anomalous origin of the left coronary artery from the pulmonary artery. $a-c$ Coronal maximum intensity projection (a), oblique axial multiplanar reformation $(b)$ and left anterior volume rendering $(c)$ images demonstrate that the left coronary artery (arrows) arises from the pulmonary trunk and is dilated, especially proximally.

Fig. 4 7-year-old boy with anomalous origin of the right coronary artery from improper aortic sinus. a-c Axial maximum intensity projection with embedded vessel cross sections $(a)$, anterior volume rendering $(b)$ and angioscopic-like $(c)$ images demonstrate that both the right (arrows) and left (curved arrows) coronaries arise from the left aortic sinus. In detail, the right coronary artery has iuxta-junctional and iuxta-commissural origin with interarterial course between the aortic root and pulmonary trunk as well as high-risk imaging features, namely acute takeoff angle, slit-like orifice, and elliptical lumen proximally (arrowhead), consistent with intramural segment. Vessel lumen returns circular more distally (dotted arrow).

Fig. 5 12-year-old boy with anomalous origin of the left coronary artery. $a$ - $d$ Anterior oblique $(a)$ and inferior $(b)$ volume rendering, curved planar reformation $(c)$ and axial maximum intensity projection $(d)$ images demonstrate that the left coronary artery originates from the opposite (right) sinus of Valsalva with a shared ostium (arrowheads) with the right coronary artery. The left main coronary artery also shows interarterial course (arrows).

Fig. 6 17-year-old boy with myocardial bridging. $a-b$ Oblique sagittal maximum intensity projection $(a)$ and left anterior oblique volume rendering $(b)$ images 
reconstructed at end-systole show a myocardial muscular band overlying the middle segment of the left anterior descending artery with minimal luminal narrowing (arrows). $c$ Left anterior oblique volume rendering view displays the intramuscular course of the vessel after removing the overlying myocardium (circle).

Fig. 7 1-year-old boy with coronary artery fistula. $a$ - $c$ Right posterior volume rendering $(a)$, oblique coronal $(b)$ and axial (c) maximum intensity projection views show a large fistulous communication between the right coronary artery (arrows), which is dilated, and the right ventricle at the level of the crux cordis (arrowheads).

Fig. 8 Male newborn with tetralogy of Fallot and double left anterior descending artery. $a-d$ Anterior $(a)$ and posterior $(b)$ volume rendering, oblique axial $(c)$ and oblique coronal $(d)$ maximum intensity projection images demonstrate a longer left anterior descending artery (arrows) arising from the right coronary (arrowheads) and coursing anteriorly to the stenotic right ventricular outflow tract (asterisk). There is also a shorter left anterior descending artery (curved arrows) coming off the left coronary (dotted arrows) and interrupting proximally but giving rise to diagonal branches.

Fig. 9 Female newborn with transposition of the great arteries and anomalous origin of the left coronary artery. $a-b$ Anterior volume rendering $(a)$ and coronal maximum intensity projection $(b)$ images show origin of the aorta from the right ventricle (asterisks) and high take-off of the left coronary artery, with likely proximal intramural course (arrows).

Fig. 10 7-year-old girl with Kawasaki disease. $a-b$ Curved planar reformation $(a)$ and left anterior volume rendering $(b)$ reconstructions show a large, partially calcified aneurysm of the left main coronary artery bifurcation (arrows).

Fig. 11 4-year-old boy with Kawasaki disease. $a$-c Right anterior volume rendering ( $a$ ) and curved planar reformation views of the right $(b)$ and the left $(c)$ coronary arteries display diffusely "beaded" coronary arteries without significant obstruction (arrows).

Fig. 12 2-year-old girl with Kawasaki disease and giant aneurysms. a First-pass horizontal long-axis reformation image documents a giant aneurysm of the middle right 
coronary artery with no visible lumen (circle) but cannot differentiate between thrombus and flow artifact. $b$ Similar horizontal long-axis reconstruction at equilibrium contrast-phase confirms the finding, distinguishing between patent lumen (arrowhead) and eccentric thrombus (arrow). $c$ - $d$ Vertical long-axis (c) and mid-ventricular shortaxis (d) views at equilibrium contrast-phase also demonstrate a persistent subendocardial perfusion defect of the left ventricular mid-basal inferior wall due to ischemia/infarct (curved arrows).

Fig. 13 12-year-old girl with transposition of the great arteries after arterial switch operation. $a$ - $d$ Anterior oblique volume rendering $(a)$, curved planar reformation $(b, c)$ and axial maximum intensity projection $(d)$ views show the right (arrows) and left (arrowheads) coronary arteries are patent at the site of reimplantation. Note the typical anterior position of the pulmonary trunk (asterisks) relative to the aorta due to Lecompte maneuver.

Fig. 14 6-year-old girl with coronary complication after arterial switch operation. $a$ Posterior volume rendering image demonstrates that the dominant left circumflex artery is not opacified at the site of reimplantation (arrow). $b$ - $d$ Vertical (b) and horizontal (c) long-axis and mid-ventricular short-axis $(d)$ reconstructions of color-coded perfusion maps confirm the finding by revealing a large transmural perfusion defect (arrowheads) in the infero-lateral wall, consistent with ischemic/infarcted myocardium.

Fig. 15 7-year old boy with coronary complication after Ross operation. $a-c$ Left anterior oblique volume rendering $(a)$, oblique axial maximum intensity projection $(b)$ and curved planar reformation $(c)$ images show that the coronary arteries are patent at the site of reimplantation (curved arrows) but the proximal portion of the left anterior descending artery is suboccluded (arrows). $d$ Horizontal long-axis reconstruction documents a subendocardial perfusion defect in the septum and left ventricular apex (arrowheads).e Similar magnetic resonance-late gadolinium enhancement image in the horizontal long-axis plane demonstrates a subendocardial infarct at the same level (dotted arrows).

Fig. 16 14-year-old boy with transposition of the great arteries after arterial switch operation and stenting of the proximal left anterior descending artery. $a-b$ Curved 
planar reformation views of the stent demonstrate mild angulation of its proximal portion and focal neointimal hyperplasia (arrows), significantly reducing the caliber of the stent lumen. $c-d$ Curved planar reformation images of the stent obtained after percutaneous transluminal angioplasty show patency restoration of the stent lumen (arrowheads). 


\section{REFERENCES}

${ }^{1}$ Lederlin M, Thambo JB, Latrabe V, Corneloup O, Cochet H, Montaudon M, et al. (2011) Coronary imaging techniques with emphasis on CT and MRI. Pediatr Radiol. 41:1516-25

${ }^{2}$ Attili A, Hensley AK, Jones FD, Grabham J, DiSessa TG. (2013) Echocardiography and coronary $\mathrm{CT}$ angiography imaging of variations in coronary anatomy and coronary abnormalities in athletic children: detection of coronary abnormalities that create a risk for sudden death. Echocardiography. 30:225-33

${ }^{3}$ Mehta R, Lee K-J, Chaturvedi R, Benson L. (2008) Complications of pediatric cardiac catheterization: a review in the current era. Catheter Cardiovasc Interv. 72(2):278-85. ${ }^{4}$ Goo HW. (2015) Coronary artery imaging in children. Korean J Radiol. 16(2):23950 .

${ }^{5}$ Han BK, Lindberg J, Grant K, Schwartz RS, Lesser JR. (2011) Accuracy and safety of high pitch computed tomography imaging in young children with complex congenital heart disease. Am J Cardiol. 107(10):1541-6.

${ }^{6}$ Han BK, Rigsby CK, Leipsic J, Bardo D, Abbara S, Ghoshhajra B, et al. (2015) Computed Tomography Imaging in Patients with Congenital Heart Disease, Part 2: Technical Recommendations. An Expert Consensus Document of the Society of Cardiovascular Computed Tomography (SCCT): Endorsed by the Society of Pediatric Radiology (SPR) and the North American Society of Cardiac Imaging (NASCI). J Cardiovasc Comput Tomogr. 9(6):493-513.

${ }^{7}$ Sigal-Cinqualbre A, Lambert V, Ronhean A, Paul JF. (2011) Role of MSCT and MRI in the diagnosis of congenital heart disease. Arch Pediatr. 18(5):617-27.

${ }^{8}$ Li J, Udayasankar UK, Toth TL, Seamans J, Small WC, Kalra MK. (2007) Automatic patient centering for MDCT: effect on radiation dose. AJR Am J Roentgenol. 188(2):547-52.

${ }^{9}$ Pache G, Grohmann J, Bulla S, Arnold R, Stiller B, Schlensak C, et al. (2011) Prospective electrocardiography-triggered CT angiography of the great thoracic vessels in infants and toddlers with congenital heart disease: feasibility and image quality. Eur J Radiol. 80(3):e440-5.

${ }^{10}$ Booij R, Dijkshoorn ML, van Straten M, du Plessis FA, Budde RP, Moelker A, et al. (2016) Cardiovascular imaging in pediatric patients using dual source CT. J Cardiovasc Comput Tomogr. 10(1):13-21. 
${ }^{11}$ Han BK, Overman DM, Grant K, Rosenthal K, Rutten-Ramos S, Cook D, et al. (2013) Non-sedated, free breathing cardiac CT for evaluation of complex congenital heart disease in neonates. J Cardiovasc Comput Tomogr. 7(6):354-60.

${ }^{12}$ Kim JW, Goo HW. (2013) Coronary artery abnormalities in Kawasaki disease: comparison between CT and MR coronary angiography. Acta Radiol. 54:156-63.

${ }^{13}$ Rigsby CK, deFreitas RA, Nicholas AC, Leidecker C, Johanek AJ, Anley P, et al. (2010) Safety and efficacy of a drug regimen to control heart rate during 64-slice ECGgated coronary CTA in children. Pediatr Radiol. 40(12):1880-9.

${ }^{14}$ Mahabadi AA, Achenbach S, Burgstahler C, Dill T, Fischbach R, Knez A, et al. (2010) Safety, efficacy, and indications of beta-adrenergic receptor blockade to reduce heart rate prior to coronary CT angiography. Radiology. 257(3):614-23.

${ }^{15}$ Decramer I, Vanhoenacker PK, Sarno G, Van Hoe L, Bladt O, Wijns W, et al. (2008) Effects of sublingual nitroglycerin on coronary lumen diameter and number of visualized septal branches on 64-MDCT angiography. AJR Am J Roentgenol. 190(1):219-25.

${ }^{16}$ Hopper KD, Mosher TJ, Kasales CJ, TenHave TR, Tully DA, Weaver JS. (1997) Thoracic spiral CT: delivery of contrast material pushed with injectable saline solution in a power injector. Radiology. 205(1):269-71.

${ }^{17}$ Goo HW. (2013) Current trends in cardiac CT in children. Acta Radiol. 54(9):105562.

${ }^{18}$ Rigsby CK, Gasber E, Seshadri R, Sullivan C, Wyers M, Ben-Ami T. (2007) Safety and efficacy of pressure-limited power injection of iodinated contrast medium through central lines in children. AJR Am J Roentgenol. 188(3):726-32.

${ }^{19}$ Litmanovich D, Zamboni GA, Hauser TH, Lin PJ, Clouse ME, Raptopoulos V. (2008) ECG-gated chest CT angiography with 64-MDCT and tri-phasic IV contrast administration regimen in patients with acute non-specific chest pain. Eur Radiol. 18(2):308-17.

${ }^{20}$ Fleischmann D. (2003) High-concentration contrast media in MDCT angiography: principles and rationale. Eur Radiol. 13 Suppl 3:N39-43.

${ }^{21}$ Stenzel F, Rief M, Zimmermann E, Greupner J, Richter F, Dewey M. (2014) Contrast agent bolus tracking with a fixed threshold or a manual fast start for coronary CT angiography. Eur Radiol. 24(6):1229-38.

${ }^{22}$ Machida H, Tanaka I, Fukui R, Shen Y, Ishikawa T, Tate E, et al. (2015) Current and Novel Imaging Techniques in Coronary CT. Radiographics. 35(4):991-1010. 
${ }^{23}$ Nie P, Wang X, Cheng Z, Ji X, Duan Y, Chen J. (2012) Accuracy, image quality and radiation dose comparison of high-pitch spiral and sequential acquisition on 128-slice dual-source CT angiography in children with congenital heart disease. Eur Radiol. 22(10):2057-66.

${ }^{24}$ Koh H, Ong CC, Choo YS, Liang CR, Tan GH, Lim TC, et al. (2016) Radiation Dose and Image Quality in Pediatric Cardiac Computed Tomography: A Comparison Between Sequential and Third-Generation Dual-Source High-Pitch Spiral Techniques. Pediatr Cardiol. 37(8):1397-1403.

25 Paul J-F, Rohnean A, Elfassy E, Sigal-Cinqualbre A. (2011) Radiation dose for thoracic and coronary step-and-shoot CT using a 128-slice dual-source machine in infants and small children with congenital heart disease. Pediatr Radiol. 41(2):244-9.

${ }^{26}$ Li T, Zhao S, Liu J, Yang L, Huang Z, Li J, et al. (2017) Feasibility of high-pitch spiral dual-source CT angiography in children with complex congenital heart disease compared to retrospective-gated spiral acquisition. Clin Radiol. 72(10):864-870.

${ }^{27}$ Nakaura T, Kidoh M, Sakaino N, Utsunomiya D, Oda S, Kawahara T, et al. (2013) Low contrast- and low radiation dose protocol for cardiac CT of thin adults at 256-row CT: usefulness of low tube voltage scans and the hybrid iterative reconstruction algorithm. Int J Cardiovasc Imaging. 29(4):913-23.

${ }^{28}$ Litmanovich DE, Tack DM, Shahrzad M, Bankier AA. (2014) Dose reduction in cardiothoracic CT: review of currently available methods. Radiographics. 34(6):146989.

${ }^{29}$ Lewis MA, Pascoal A, Keevil SF, Lewis CA. (2016) Selecting a CT scanner for cardiac imaging: the heart of the matter. Br J Radiol. 89(1065):20160376.

${ }^{30}$ Jadhav SP, Golriz F, Atweh LA, Zhang W, Krishnamurthy R. (2015) CT angiography of neonates and infants: comparison of radiation dose and image quality of target mode prospectively ECG-gated 320-MDCT and ungated helical 64-MDCT. AJR Am J Roentgenol. 204(2):W184-91.

${ }^{31}$ Podberesky DJ, Angel E, Yoshizumi TT, Toncheva G, Salisbury SR, Alsip C, et al. (2012) Radiation dose estimation for prospective and retrospective ECG-gated cardiac CT angiography in infants and small children using a 320-MDCT volume scanner. AJR Am J Roentgenol. 199(5):1129-35.

${ }^{32}$ Moscariello A, Takx RAP, Schoepf UJ, Renker M, Zwerner PL, O’Brien TX, et al. (2011) Coronary CT angiography: image quality, diagnostic accuracy, and potential for 
radiation dose reduction using a novel iterative image reconstruction technique comparison with traditional filtered back projection. Eur Radiol. 21(10):2130-8.

${ }^{33}$ Abbara S, Blanke P, Maroules CD, Cheezum M, Choi AD, Han BK, et al. (2016) SCCT guidelines for the performance and acquisition of coronary computed tomographic angiography: A report of the society of Cardiovascular Computed Tomography Guidelines Committee: Endorsed by the North American Society for Cardiovascular Imaging (NASCI). J Cardiovasc Comput Tomogr. 10(6):435-49.

${ }^{34}$ Leipsic J, Abbara S, Achenbach S, Cury R, Earls JP, Mancini GJ, et al. (2014) SCCT guidelines for the interpretation and reporting of coronary CT angiography: a report of the Society of Cardiovascular Computed Tomography Guidelines Committee. J Cardiovasc Comput Tomogr. 8(5):342-58.

${ }^{35}$ Karlo CA, Leschka S, Stolzmann P, Glaser-Gallion N, Wildermuth S, Alkadhi H. (2012) A systematic approach for analysis, interpretation, and reporting of coronary CTA studies. Insights Imaging. 3(3):215-28.

${ }^{36}$ Zhang LJ, Zhou CS, Wang Y, Jin Z, Yu W, Zhang Z, et al. (2014) Prevalence and types of coronary to pulmonary artery fistula in a Chinese population at dual-source CT coronary angiography. Acta Radiol. 55(9):1031-9.

${ }^{37}$ Chao BT, Wang XM, Wu LB, Chen J, Cheng ZP, Wu DW, et al. (2012) Diagnostic value of dual-source CT in Kawasaki disease. Chin Med J (Engl). 123(6):670-4.

${ }^{38}$ Neves PO, Andrade J, Monção H. (2015) Coronary anomalies: what the radiologist should know. Radiol Bras. 48(4):233-41.

${ }^{39}$ Pursnani A, Jacobs JE, Saremi F, Levisman J, Makaryus AN, Capuñay C, et al. (2012) Coronary CTA assessment of coronary anomalies. J Cardiovasc Comput Tomogr. 6(1):48-59.

40 Secinaro A, Ntsinjana H, Tann O, Schuler PK, Muthurangu V, Hughes M, et al. (2011) Cardiovascular magnetic resonance findings in repaired anomalous left coronary artery to pulmonary artery connection (ALCAPA). J Cardiovasc Magn Reson. $16 ; 13: 27$.

${ }^{41}$ Agarwal PP, Dennie C, Pena E, Nguyen E, LaBounty T, Yang B, et al. (2017) Anomalous Coronary Arteries That Need Intervention: Review of Pre- and Postoperative Imaging Appearances. Radiographics. 37(3):740-57.

${ }^{42}$ Kim SY, Seo JB, Do KH, Heo JN, Lee JS, Song JW, et al. (2006) Coronary artery anomalies: classification and ECG-gated multi-detector row CT findings with angiographic correlation. Radiographics. 26(2):317-33. 
43 Alegria JR, Herrmann J, Holmes DR, Lerman A, Rihal CS. (2005) Myocardial bridging. Eur Heart J. 26(12):1159-68.

${ }^{44}$ Hwang JH, Ko SM, Roh HG, Song MG, Shin JK, Chee HK, et al. (2010) Myocardial bridging of the left anterior descending coronary artery: depiction rate and morphologic features by dual-source CT coronary angiography. Korean J Radiol. 11(5):514-21.

${ }^{45}$ Ishikawa Y, Akasaka Y, Suzuki K, Fujiwara M, Ogawa T, Yamazaki K, et al. (2009) Anatomic properties of myocardial bridge predisposing to myocardial infarction. Circulation. 120(5):376-83.

${ }^{46}$ Husmann L, Nkoulou R, Wolfrum M, Kaufmann PA. (2011) Myocardial bridging causing infarction and ischaemia. Eur Heart J. 32(7):790.

${ }^{47}$ Said SA, el Gamal MI, van der Werf T. (1997) Coronary arteriovenous fistulas: collective review and management of six new cases - changing etiology, presentation, and treatment strategy. Clin Cardiol. 20:748-52.

${ }^{48}$ Yu FF, Lu B, Gao Y, Hou ZH, Schoepf UJ, Spearman JV, et al. (2013) Congenital anomalies of coronary arteries in complex congenital heart disease: diagnosis and analysis with dual-source CT. J Cardiovasc Comput Tomogr. 7(6):383-90.

${ }^{49}$ Han BK, Rigsby CK, Hlavacek A, Leipsic J, Nicol ED, Siegel MJ, et al. (2015) Computed Tomography Imaging in Patients with Congenital Heart Disease Part I: Rationale and Utility. An Expert Consensus Document of the Society of Cardiovascular Computed Tomography (SCCT): Endorsed by the Society of Pediatric Radiology (SPR) and the North American Society of Cardiac Imaging (NASCI). J Cardiovasc Comput Tomogr. 9(6):475-92.

${ }^{50}$ Agarwal PP, Kazerooni EA. (2008) Dual left anterior descending coronary artery: CT findings. AJR Am J Roentgenol. 191(6):1698-701.

${ }^{51}$ Warnes CA, Williams RG, Bashore TM, Child JS, Connolly HM, Dearani JA, et al. (2008) ACC/AHA 2008 guidelines for the management of adults with congenital heart disease: a report of the American College of Cardiology/American Heart Association Task Force on Practice Guidelines (writing committee to develop guidelines on the management of adults with congenital heart disease). Circulation. 118(23):e714-833. 52 Sithamparanathan S, Padley SP, Rubens MB, Gatzoulis MA, Ho SY, Nicol ED. (2013) Great vessel and coronary artery anatomy in transposition and other coronary anomalies: a universal descriptive and alphanumerical sequential classification. JACC Cardiovasc Imaging. 6(5):624-30. 
${ }^{53}$ Swanson SK, Sayyouh MM, Bardo DME, Ghadimi Mahani M, Lu JC, Dorfman AL, et al. (2018) Interpretation and Reporting of Coronary Arteries in Transposition of the Great Arteries: Cross-sectional Imaging Perspective. J Thorac Imaging. 33(4):W14W21.

${ }^{54}$ Legendre A, Losay J, Touchot-Koné A, Serraf A, Belli E, Piot JD, et al. (2003) Coronary events after arterial switch operation for transposition of the great arteries. Circulation. 108 Suppl 1:II186-190.

${ }^{55}$ Newburger JW, Takahashi M, Gerber MA, Gewitz MH, Tani LY, Burns JC, et al. (2004) Diagnosis, treatment, and long-term management of Kawasaki disease: a statement for health professionals from the Committee on Rheumatic Fever, Endocarditis and Kawasaki Disease, Council on Cardiovascular Disease in the Young, American Heart Association. Circulation. 110(17):2747-71.

${ }^{56}$ Suda K, Iemura M, Nishiono H, Teramachi Y, Koteda Y, Kishimoto S, et al. (2011) Long-term prognosis of patients with Kawasaki disease complicated by giant coronary aneurysms: a single-institution experience. Circulation. 123(17):1836-42.

${ }^{57}$ Kato H, Sugimura T, Akagi T, Sato N, Hashino K, Maeno Y, et al. (1996) Long-term consequences of Kawasaki disease. A 10- to 21-year follow-up study of 594 patients. Circulation. 94(6):1379-85.

${ }^{58}$ Yu Y, Sun K, Wang R, Li Y, Xue H, Yu L, et al. (2011) Comparison study of echocardiography and dual-source CT in diagnosis of coronary artery aneurysm due to Kawasaki disease: coronary artery disease. Echocardiography. 28:1025-34.

${ }^{59}$ Arnold R, Ley S, Ley-Zaporozhan J, Eichhorn J, Schenk JP, Ulmer H, et al. (2007) Visualization of coronary arteries in patients after childhood Kawasaki syndrome: value of multidetector $\mathrm{CT}$ and MR imaging in comparison to conventional coronary catheterization. Pediatr Radiol. 37(10):998-1006.

${ }^{60}$ Gaca AM, Jaggers JJ, Dudley LT, Bisset GS 3rd. (2008) Repair of congenital heart disease: a primer-part 1. Radiology. 247(3):617-31.

${ }^{61}$ Bonhoeffer P, Bonnet D, Piéchaud JF, Stümper O, Aggoun Y, Villain E, et al. (1997) Coronary artery obstruction after the arterial switch operation for transposition of the great arteries in newborns. J Am Coll Cardiol. 29(1):202-6.

${ }^{62}$ Ou P, Celermajer DS, Marini D, Agnoletti G, Vouhé P, Brunelle F, et al. (2008) Safety and accuracy of 64-slice computed tomography coronary angiography in children after the arterial switch operation for transposition of the great arteries. JACC Cardiovasc Imaging. 1(3):331-9. 
${ }^{63}$ Laudito A, Brook MM, Suleman S, Bleiweis MS, Thompson LD, Hanley FL, et al. (2001) The Ross procedure in children and young adults: a word of caution. J Thorac Cardiovasc Surg. 122(1):147-53.

${ }^{64}$ Eichhorn JG, Jourdan C, Hill SL, Raman SV, Cheatham JP, Long FR. (2008) CT of pediatric vascular stents used to treat congenital heart disease. Am J Roentgenol. 190(5):1241-6.

${ }^{65}$ Rohnean A, Houyel L, Sigal-Cinqualbre A, To NT, Elfassy E, Paul JF. (2011) Heart transplant patient outcomes: 5-year mean follow-up by coronary computed tomography angiography. Transplantation. 91:583-8.

${ }^{66}$ Araoz PA, Kirsch J, Primak AN, Braun NN, Saba O, Williamson EE, et al. (2009) Optimal image reconstruction phase at low and high heart rates in dual-source CT coronary angiography. Int J Cardiovasc Imaging. 25:837-45.

${ }^{67}$ Leipsic J, LaBounty TM, Ajlan AM, Earls JP, Strovski E, Madden M, et al. (2013) A prospective randomized trial comparing image quality, study interpretability, and radiation dose of narrow acquisition window with widened acquisition window protocols in prospectively ECG-triggered coronary computed tomography angiography. J Cardiovasc Comput Tomogr. 7(1):18-24.

${ }^{68}$ Pan C-J, Qian N, Wang T, Tang X-Q, Xue Y-J. (2013) Adaptive prospective ECGtriggered sequence coronary angiography in dual-source CT without heart rate control: Image quality and diagnostic performance. Exp Ther Med. 5(2):636-42. 